\title{
Child-Pugh Class A
}

National Cancer Institute

\section{Source}

National Cancer Institute. Child-Pugh Class A. NCI Thesaurus. Code C113691.

Child-Pugh score indicating one-year survival of $100 \%$ in patients with chronic liver disease and cirrhosis. This score is determined by the study of the following five factors:

bilirubin, albumin, international normalized ratio, presence and degree of ascites, and presence and degree of encephalopathy. 